\title{
Caracterización mecánica de bioesponjas de residuos de crustáceos como opción al poliestireno expandido en empaques para alimentos
}

\author{
JESÚS A. ORTEGA-GRANADOS', ROLANDO S. GARCÍA-GÓMEZI \\ RONNY FLORES ${ }^{2}$, MARÍA DEL CARMEN DURÁN-DOMIINGUEZ-DE-BAZÚA ${ }^{1 *}$ \\ 'Laboratorios 301, 302, 303 de Ingeniería Química Ambiental y de Química Ambiental, Facultad de Química, \\ UNAM. 2Laboratorio de Química Ambiental y Sostenible, \\ Facultad de Ciencias Químicas, Universidad Central del Ecuador \\ *Correspondencia: mcduran@unam.mx
}

Recibido: 06 de enero de 2015, Aceptado: 04 de agosto de 2015

\section{Resumen}

Se propone aprovechar los subproductos de las industrias de los crustáceos, tomando como ejemplo a la industria camaronera, ya que contienen cantidades interesantes del biopolímero quitina, que podría usarse para producir envases desechables para alimentos, similares a los de poliestireno expandido, PE, utilizando una metodología amigable con el ambiente y empleando una mezcla de aditivos de grado alimentario: una mezcla de carboximetilcelulosa, a tres diferentes concentraciones (10,20,30\%) y almidón de tapioca (Manihot esculenta), con una concentración, $40 \%$. Se midió una propiedad mecánica de tracción (compresión) usando PE como blanco para las bioesponjas. Se obtuvieron resultados mejores para las probetas experimentales de quitina-quitosana ( $10 \%$ de carboximetilcelulosa y $40 \%$ almidón de tapioca) en comparación con el control de poliestireno expandido: Módulos de Young de $0.5087 \pm 0.061$ y $0.535 \pm 0.065 \mathrm{MPa}$ versus 7.832 y 1.858 para el PE de densidad de $0.035 \mathrm{~g} / \mathrm{cm}^{3}$ y 4.555 y $4.546 \mathrm{MPa}$ para el de $0.018 \mathrm{~g} / \mathrm{cm}^{3}$ de densidad, respectivamente. Esto indica que son menos deformables que el poliestireno espumado. Los resultados son alentadores y deben ser corroborados con la producción de bandejas reales.

Palabras clave: empaques de alimentos, poliestireno espumado, bioesponjas de quitina, residuos sólidos de crustáceos.

\section{Mechanical characterization of bio-sponges from crustacean wastes as an option for expanded polystyrene in food packaging}

\section{Abstract}

The use of crustacean byproducts is proposed and shrimp residues are use as an example, since they contain interesting amounts of chitin, that might be used to produce disposable packaging for foods, similar to those of expanded polystyrene, $P E$, utilizing an environmentally friendly methodology and using a mixture of GRAS additives: carboxymethylcellulose, at three different concentrations (10,20,30\%) and tapioca starch (Manihot esculenta), with a concentration of $40 \%$. A traction mechanical property (compression) using PE as blank for the biosponges was measured. Better results were obtained for the experimental specimens with $10 \%$ carboximethylcellulose and $40 \%$ tapioca starch in comparison with the expanded polystyrene, PE: Modules of Young of $0.5087 \pm 0.061$ and $0.535 \pm 0.065 \mathrm{MPa}$ versus 7.832 and 1.858 for the PE with density of $0.035 \mathrm{~g} / \mathrm{cm}^{3}$ and 4.555 and $4.546 \mathrm{MPa}$ for the PE with density of $0.018 \mathrm{~g} / \mathrm{cm}^{3}$, respectively. These data indicate that biosponges are less deformable than expanded polystyrene. Results are encouraging and should be corroborated with real tests using trays used for containing foods.

Key words: food packaging, expanded polystyrene from oil derivatives, chitin biosponges, crustacean solid residues. 


\section{Introducción}

La intensificación de la industrialización que se presentó en México durante la segunda mitad del siglo pasado, produjo una mayor demanda de materias primas para satisfacer el creciente consumo de bienes y servicios de una población en aumento y con patrones de consumo cambiantes y cada vez más demandantes [1]. El Instituto Nacional de Estadística y Geografía reporta que en México se produjeron 40 millones de toneladas de basura: Residuos orgánicos (52.4\%), plásticos y vidrios $(16.8 \%)$, productos de papel y cartón $(13.8 \%)$ y metales $(3.4 \%)$. De estos residuos sólidos, el $62.2 \%$ del total tiene disposición final en rellenos sanitarios y el $25.3 \%$ en tiraderos a cielo abierto [2]. Respecto de los plásticos en México, en el año 2002, el consumo fue de 1.4 millones de toneladas [3]. Cuando no son desechados adecuadamente, pueden causar la muerte de algunos animales que los confunden con comida, como ha ocurrido con las tortugas y aves marina. También puede suceder que se quedan atrapados en ellos, como es el caso de algunas aves que se enredan en los arillos de plástico usados para empacar latas de aluminio.

El camarón, un crustáceo muy apreciado, se encuentra posicionado por su masa en el segundo lugar de la producción pesquera en México. Por su valor económico, está en el primer lugar. La tasa media de crecimiento anual de la producción en los últimos 10 años es de $6.24 \%$, lo cual se debe al crecimiento de la actividad acuícola de dicha especie. En las exportaciones se encuentra en el primer lugar de las especies pesqueras, siendo Estados Unidos, Japón y Francia sus principales destinos. La producción de camarón en México en el 2011 fue de 184,123 Ton (biomasa completa) representando un valor económico en 513,121 dólares estadounidenses. Los estados mexicanos con mayor participación porcentual de la producción de camarón fueron Sinaloa $(42.92 \%)$, Sonora $(28.47 \%)$, Nayarit (8.83\%) y Tamaulipas (6.31\%) [4].

Los camarones son decápodos de agua dulce o salada con infraorden Caridea. Siendo un producto alimenticio, solamente el $50 \%$ es comestible, dejando el $50 \%$ restante como un subproducto de alto valor por su contenido de quitina y otros productos útiles [5]. La composición del cefalotórax del camarón muestra que puede ser aprovechado ya que está compuesto, en base seca, de $44.7 \%$ de proteína, de $26.3 \%$ de ceniza, $20.7 \%$ de fibra cruda, $5.2 \%$ de grasas y $3.1 \%$ de otros hidratos de carbono. De esta composición bromatológica destaca la quitina con 14 a $27 \%$ [5-6].

Estimando que el $50 \%$ de la producción se traduce en subproductos sólidos, re-aprovechables utilizando el cefalotórax y exoesqueleto como materia prima para producir quitina, en México se cuenta con aproximadamente el $50 \%$ de las 184,123 toneladas anuales, dando 92,061.5 Ton anuales. El cefalotórax y exoesqueleto tienen una función de protección, respiratoria y mecánica en los artrópodos [7-1 1]. Los residuos generados durante el descabezado y pelado del camarón pueden, por tanto, ser una fuente de recursos muy importante, especialmente los pigmentos y la quitina [12].

La quitosana, goma derivada químicamente de la desacetilación de la quitina, comparte con ella algunas propiedades en común entre las que se destacan: adsorción, actividad antimicrobiana, biodegradabilidad, capacidad para soportar mucha fuerza de tensión, flexibilidad y fácil modificación $[9,13]$. Su aplicación ha tenido resultados favorables en la ingeniería ambiental. Se ha usado en el tratamiento de aguas residuales, en la adsorción de $\mathrm{Cr}$ (III) de efluentes contaminados [14]. La quitina, a pesar de su insolubilidad en agua y muchos disolventes, tiene el potencial para formar una esponja estable altamente insoluble en agua y biodegradable $[5,6,15,16]$.

En esta fase de la investigación se propone aprovechar la producción de desechos de la industria camaronera, siendo una de las industrias más importantes en México ya que poseen cantidades interesantes de quitina. Con estos desechos podría disminuirse la generación de residuos de plásticos derivados del petróleo, como los envases desechables de la comida rápida fabricados con poliestireno expandido, utilizando una metodología amigable con el ambiente basada en la química verde. En ella se extrae una mezcla de quitina-quitosana de los desechos de cefalotórax y exoesqueletos de camarón produciendo una bioesponja, especialmente si se le agregan mezclas de aditivos de grado alimentario, buscando obtener una bioesponja de quitina-quitosana con propiedades mecánicas similares o mejores que las del poliestireno expandido. 
El objetivo fue corroborar la bondad de la adición de dos agentes plastificantes a la quitina-quitosana obtenida de los residuos de crustáceos para darles propiedades similares a las de sus contrapartes derivadas del petróleo usando para ello una prueba de compresión y la medición del Módulo de Young en un equipo Instron y en uno de pruebas triaxiales cíclicas [17-20]. La meta última es que sean estables a condiciones ambientales $y$ fácilmente biodegradables en rellenos sanitarios.

\section{Parte experimental}

\subsection{Tratamiento del cefalotórax de cama- rón fresco y elaboración del disolvente MAC- $141^{\circ}$}

Mediante un proceso basado en la química verde para la extracción de la quitina se empleó un disolvente amigable con el ambiente patentado en los Laboratorios 301, 302 y 303 de la Facultad de Química de la UNAM [21]. En la literatura se presenta la metodología precisa para tratar el cefalotórax y para obtener este disolvente $[5,6]$. El disolvente es una mezcla de agua, metanol y cloruro de calcio, patentado como MAC-141@ $[7,8,21]$. En investigaciones previas $[5,6,22]$ se desarrollaron las metodologías para la extracción de quitina y, por el propio proceso, su derivado desacetilado, la quitosana. La extracción se llevó a cabo en un reactor con una mezcla de residuos sólidos de camarón y el disolvente $M A C-141^{\circ}$ en una relación 1:10, respectivamente, para la obtención de la mezcla de quitina-quitosana (Q-Qn) disuelta en el disolvente MAC-141 ${ }^{\odot}$. La extracción de la quitina-quitosana con el disolvente MAC-141@ permitió obtener una solución de quitina y sólidos de los componentes de la harina de residuos de camarón $[7,8,22]$. Los sólidos se separaron por filtración con vacío en un equipo Millipore empleando como medio filtrante una rejilla de plástico. La descripción detallada está en la literatura $[7,8]$.

El diagrama de bloques de la adecuación de la metodología se encuentra en proceso de patentamiento [23]. Para la obtención de las bioespumas de los polímeros naturales quitina-quitosana $\left(Q_{-}-Q_{n}\right)$ a temperatura ambiente $\left(20 \pm 2^{\circ} \mathrm{C}\right)$, se utilizó la metodología reportada $[5,6,20,24,25]$, usando solamente el disolvente MAC-141.

\subsection{Producción de bioesponjas}

Posteriormente, la solución extraída se vertió en cajas de Petri de vidrio, las cuales se colocaron en una cámara con la atmósfera saturada de agua para que el calcio del disolvente MAC(C) presente en la película se solubilizara y, de esta manera, poder retirar la mayor cantidad de iones calcio del polímero de quitina. Después de la cámara de humidificación, en el proceso se realizó una operación unitaria de agitación para la formación de la bioespuma (adicionando dos agentes plastificantes, carboximetilcelulosa, CMC, y almidón de tapioca, AlmTap.

\subsubsection{Elaboración de las probetas de las bioespumas usadas para las pruebas mecánicas}

Se prepararon las probetas de las bioespumas a las diferentes concentraciones de agentes plastificantes de acuerdo con la Tabla 1, empleando soluciones de quitosana Sigma Aldrich (85\% desacetilación) disuelta en disolvente MAC-141 ${ }^{\circ}$. Estas se vertieron en vasos de precipitados de $250 \mathrm{~mL}$ de capacidad con agitación continua para homogeneizar y adicionar aire para la formación de la espuma. Con las bioespumas se prepararon probetas cilíndricas de $36 \mathrm{~mm}$ (diámetro) x $75 \mathrm{~mm}$ (altura). Dichas probetas se cortaron con un "suaje" o molde y se tomaron medidas de la anchura y el espesor con un Vernier. Posteriormente, cada probeta se sometió a pruebas mecánicas en dos equipos mencionados en la literatura [7], uno de ellos de la marca Instron. Las pruebas mecánicas se aplicaron para evaluar la bondad del biopolímero obtenido y de los dos agentes plastificantes con objeto de que sus propiedades lleguen a ser prácticamente idénticas a las del poliestireno espumado.

Tabla 1. Comparación entre las bioespumas en estudio obtenidas del cefalotoráx y exoesqueleto de camarón con un disolvente amigable con el ambiente (MAC-1410) y un control (poliestireno expandido, $P E$, unicel)

\begin{tabular}{|c|c|c|}
\hline \multirow{2}{*}{ Película } & \multicolumn{2}{|c|}{$\begin{array}{c}\text { Agentes plastificantes biodegradables } \\
\text { (APB) }\end{array}$} \\
\hline \multirow{4}{*}{ Q-Qn } & $\begin{array}{c}\text { Carboximetilcelulosa } \\
\text { CMC } \\
{[\%]}\end{array}$ & $\begin{array}{c}\text { Almidón de tapioca } \\
\text { AlmTap } \\
{[\%]}\end{array}$ \\
\cline { 2 - 3 } & 10.0 & 40.0 \\
\cline { 2 - 3 } & 20.0 & 40.0 \\
\cline { 2 - 3 } & 30.0 & 40.0 \\
\hline Control & \multicolumn{2}{|c|}{ Poliestireno expandido (unicel) } \\
\hline
\end{tabular}




\subsubsection{Pruebas mecánicas de las bioespumas}

Las pruebas mecánicas que se realizaron a las bioespumas fueron mediante dos métodos de compresión simple. En ellos se vio el comportamiento mecánico de las probetas y se compararon con probetas similares de poliestireno expandido (unicel). Los equipos en que se realizaron las pruebas fueron: a) un equipo Instron Modelo Series IX (Automated Materials Testing System) y b) un equipo de compresión triaxial estática simple no confinada construido en el Instituto de Ingeniería de la UNAM, en el Laboratorio de Suelos.

\subsubsection{Método estándar de propiedades de compresión para plásticos celulares rígidos ASTM D 1621-00 [7]}

Con las bioespumas que presentaron una mayor dureza, determinada de manera subjetiva (visual y táctil) se prepararon las probetas cilíndricas de $36 \mathrm{~mm}$ (diámetro) $\times 75 \mathrm{~mm}$ (altura). Las pruebas mecánicas que se aplicaron para evaluar la bondad del biopolímero obtenido con los dos agentes plastificantes buscando que sus propiedades lleguen a ser prácticamente idénticas a las del poliestireno espumado siguieron los estándares internacionales de comportamiento esfuerzo-deformación para obtener el valor del Módulo de Young, E.

\subsubsection{Método estándar para suelos cohesi- vos tipo no consolidado y no drenado ASTM [7]}

Esta norma tiene como objetivo determinar la resistencia al esfuerzo cortante y la relación esfuerzo-deformación de una muestra cilíndrica de suelo cohesivo inalterada o remoldeada. Es una prueba lenta, es decir, el espécimen recibe un esfuerzo de compresión en pequeños incrementos, esperando siempre que en cada incremento el valor sea cero, para que el material se consolide y que la presión interna sea cero. Estas pruebas son de mayor confiabilidad al momento de determinar la resistencia de materiales porosos como el suelo y dan la opción de conocer en forma más completa las características mecánicas del material.

\subsubsection{Espectroscopía infrarroja por trans- formada de Fourier método ASTM [7]}

El método de detección tiene una resolución de $4 \mathrm{~cm}^{-1}$. El equipo está acoplado con un microscopio óptico de análisis infrarrojo y de luz visible. El modo de preparación de la muestra necesita que éstas sean individuales.

\subsubsection{Microscopía de barrido electrónico para las bioespumas o bioesponjas}

El microscopio electrónico de barrido de bajo vacío MEBBV (SEM-LV, por sus siglas en inglés Scanning Electron Microscope-Low Vaccum) es usado para observar especímenes no conductores, que contienen relativamente poca agua y que se deforman al ser procesados de manera convencional. Este equipo permite observarlos directamente en modo de bajo vacío, sin tener que deshidratarlos o recubrirlos con material conductor, lo que evita la introducción de artefactos por el procesamiento [26].

\section{Resultados y discusión}

En esta investigación se obtuvieron rendimientos de hasta casi $40 \%$ en masa de harina parcialmente desproteinizada de cefalotórax y exoesqueletos con respecto a los residuos totales (Tabla 2). La extracción de la quitina se llevó a cabo a temperatura ambiente $\left(20 \pm 2^{\circ} \mathrm{C}\right)$ y sin el uso de sustancias tóxicas ni agresivas con la molécula de quitina ni con el medio y por ello se le considera amigable con el ambiente [27]. En la Tabla 2 se observa que el rendimiento para el lote de $2.20 \mathrm{~kg}$ de cefalotórax y exoesqueletos de camarón frescos fue de $27.27 \%$, obteniéndose $0.600 \mathrm{~kg}(600 \mathrm{~g})$ de harina de camarón. El rendimiento mayoritario lo presenta el lote de $2.0 \mathrm{~kg}$ de cefalotórax y exoesqueletos de camarón frescos, con un rendimiento del $37.50 \%$. Esto significa que hasta el $37 \%$ del total que es considerado como desperdicio, aún puede ser recuperado y aprovechado para la extracción de quitina y la elaboración de esponjas [24,25]. Las diferencias en los rendimientos se deben a que, proviniendo de la "Central de Abastos" de la Ciudad de México, las bolsas tenían muchos desperdicios o restos de otras especies marinas (como pulpo, cabezas de pescado y estrellas de mar).

Tabla 2. Porcentaje de harina recuperada a partir de camarón: cefalotórax y exoesqueletos frescos

\begin{tabular}{ccc}
\hline $\begin{array}{c}\text { Cefalotórax y } \\
\text { exoesqueletos } \\
\text { de camarón } \\
{[\mathrm{kg}]^{*}}\end{array}$ & $\begin{array}{c}\text { Harina de residuos } \\
\text { de } \\
\text { camarón }[\mathrm{kg}]^{*}\end{array}$ & $\begin{array}{c}\text { Porcentaje de } \\
\text { harina } \\
\text { recuperada } \\
{[\%]}\end{array}$ \\
\hline 2.20 & 0.600 & 27.27 \\
\hline 2.10 & 0.680 & 32.38 \\
\hline 2.00 & 0.750 & 37.50 \\
\hline 2.15 & 0.710 & 33.02 \\
\hline
\end{tabular}

* b.h. Base húmeda 


\subsection{Eficiencia del disolvente MAC- $141^{\odot}$ para la extracción de la mezcla de quitina- quitosana (Q-Qn) de los residuos secos de camarón}

De acuerdo con lo reportado por Flores en 2004 y 2008 $[5,6,21]$, el calcio que no pudo solubilizarse en el disolvente MAC- $141^{\circ}$ por el efecto de la solvatación del calcio en el metanol y agua puede ser recuperado, como se realizó en esta investigación y ser regresado al proceso de obtención del disolvente MAC-1 $41^{\circ}$ debido a que en una mayor concentración de calcio genera una mayor extracción de bioespumas (Tabla 3).

La Tabla 4 presenta el balance de masa para la extracción de quitina de la harina de camarón. De acuerdo a los resultados obtenidos el método permite que lo que es considerado como desperdicio, pueda ser recuperado y aprovechado para la extracción de quitina y la elaboración de esponjas que podrían ser útiles para la elaboración de recipientes de un solo uso $[5,6]$. La Figura 1 presenta la formación de bioespuma quitina.

Tabla 3. Rendimiento en la recuperación de calcio del disolvente MAC-14 $1^{\odot}$

\begin{tabular}{|c|c|c|c|c|c|c|}
$\begin{array}{c}\text { Cloruro de calcio } \\
\text { comercial } \\
\text { [g] }\end{array}$ & $\begin{array}{c}\text { MeOH } \\
{[\mathbf{m L}]}\end{array}$ & $\begin{array}{c}\mathbf{H}_{2} \text { O llave o } \\
\text { grifo } \\
{[\mathbf{m L}]}\end{array}$ & $\begin{array}{c}\text { MAC } \\
\text { obtenido } \\
\text { [g] }\end{array}$ & $\begin{array}{c}\text { Residuos de calcio } \\
\text { y agua) } \\
\text { [g] }\end{array}$ & $\begin{array}{c}\text { Calcio } \\
\text { recuperado }\end{array}$ & $\begin{array}{c}\text { Porcentaje de } \\
\text { calcio recuperado }\end{array}$ \\
\hline $\mathbf{2 7 7 . 4 5}$ & 99 & 180 & 398 & 93.18 & 58.45 & 21.07 \\
\hline $\mathbf{2 7 7 . 4 5}$ & 99 & 180 & 335 & 87.58 & 60.54 & 21.82 \\
\hline $\mathbf{2 7 7 . 4 5}$ & 99 & 180 & 420 & 68.86 & 36.57 & 13.18 \\
\hline $\mathbf{2 7 7 . 4 5}$ & 99 & 180 & 420 & 91.06 & 37.82 & 13.63 \\
\hline $\mathbf{2 7 7 . 4 5}$ & 99 & 180 & 476 & 76.00 & 56.44 & 20.34 \\
\hline
\end{tabular}

Tabla 4. Balances de masa de las extracciones de harinas secas de cefalotórax y exoesqueleto de camarón usando el disolvente MAC-141@ después de 48 horas a temperatura ambiente $\left(20 \pm 2^{\circ} \mathrm{C}\right)$ para obtener las bioespumas o bioesponjas

\begin{tabular}{|c|c|c|c|c|c|c|c|c|}
\hline Muestra & \multicolumn{5}{|c|}{ Inicio } & \multicolumn{2}{c|}{ Después de 48 horas } & \multicolumn{2}{c|}{ Esponja de quitina obtenida } \\
por filtración
\end{tabular}




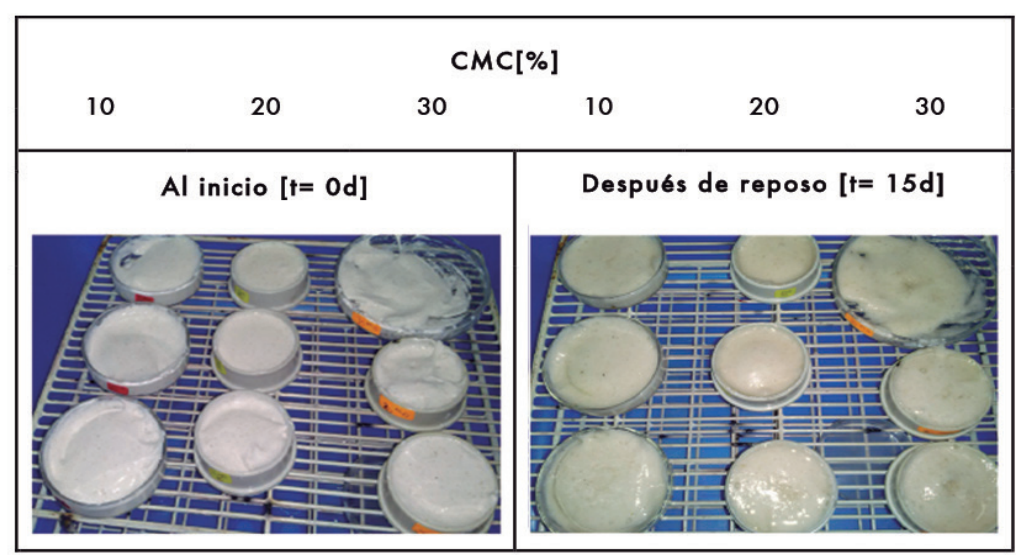

Figura 1. Bioespumas con la mezcla de los dos agentes plastificantes, CMC y $40 \%$ de almidón de tapioca

\subsection{Caracterización de las bioespumas 0 bioesponjas de quitina-quitosana (Q-Qn) adicionadas con agentes plastificantes}

De acuerdo con la obtención de las mejores concentraciones de los agentes plastificantes se identificaron las propiedades estructurales de las bioespumas, mediante técnicas analíticas como se describen a continuación y que permitieron definir según su estructura cuáles serían las mejores candidatas para realizar las pruebas mecánicas mediante las dos metodologías descritas anteriormente. A continuación se presentan los resultados de la microscopía electrónica de barrido y los espectros de FT-IR para las probetas del material en estudio.

\subsubsection{Microscopía electrónica de barrido para las bioespumas}

En la literatura, reportado por Núñez-Bretón en 2009 [28] se menciona que la incorporación del almidón produce una película plástica con una estructura porosa, permitiendo el acceso entre las moléculas plásticas al oxígeno, así bien se muestra en la Figura 1, donde las mezclas de bioespuma de Q-Qn con los agentes plastificantes 10, 20 y $30 \%$ CMC, se vieron favorecidas por el $40 \%$ de almidón de tapioca, que se encontraba permitiendo la inclusión de aire observándose los poros dentro del material diseñado.
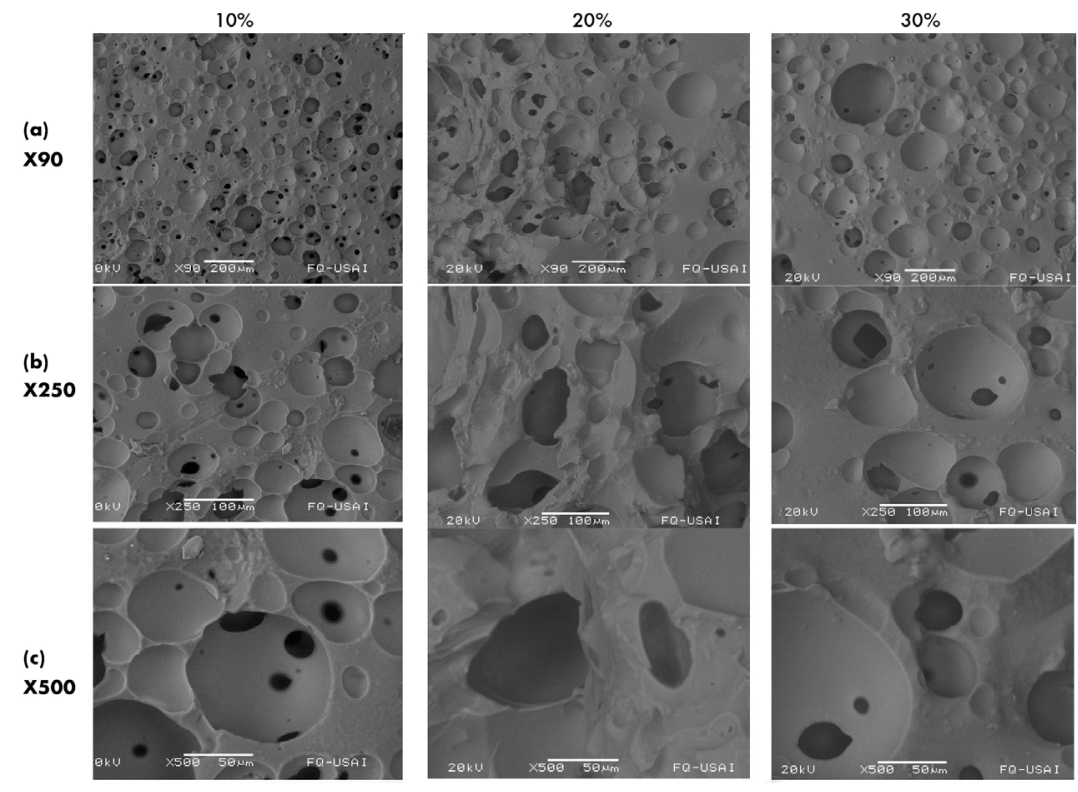

Figura 2. Microscopía electrónica de barrido de bioespumas o bioesponjas de quitina-quitosana, Q-Qn, con la mezcla de agentes plastificantes al 10,20 y 30\% de carboximetilcelulosa, CMC, y de 40\% almidón de tapioca, Alm-Tap, en ellas. 
En la Figura 2 se presentan las microfotografías obtenidas con ayuda del equipo de microscopía electrónica de barrido, donde se observa la fractura o falla de la bioespuma de Q-Qn con la mezcla de agentes plastificantes 10,20 y $30 \%$ CMC y $40 \%$ almidón de tapioca, sometida con los equipos de compresión simple para determinar el Módulo de Young (E), el uso de almidón podría ser considerado como composta en un relleno sanitario por su biodegradabilidad. En la literatura, lo reportado por Flores en 2004 y 2008 [5,6] menciona que la estructura de la quitina (Figura 3.a) presenta una diferencia con la forma de celda entre la espuma de poliestireno, poliuretano y el material en estudio. La espuma de poliestireno presenta celdas hexagonales (Figura 3.b) y la espuma de poliuretano es la que más se asemeja a la muestra en estudio presentando celdas abiertas deformes (Figura 3.c).
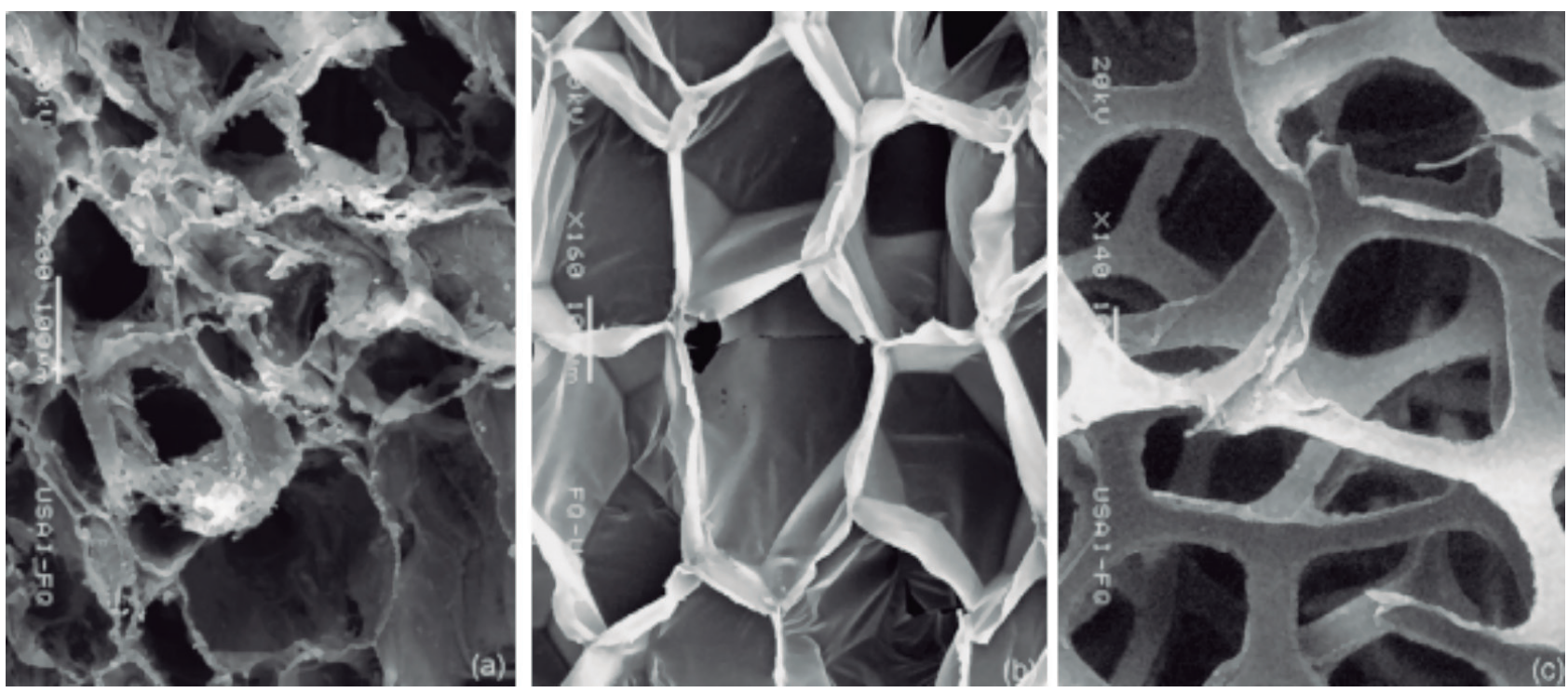

Figura 3. Microscopía electrónica de barrido de: (a) esponja de quitina, (b) espuma de poliestireno y (c) espuma de poliuretano. Tomada de Flores (2008) [6]

En la Figura 4 se muestran los resultados, analizados también mediante microscopía electrónica de barrido, de los materiales usados en esta investigación, especialmente de la carboximetilcelulosa grado alimenticio, CMC, ya que en la literatura se ha encontrado que es un formador de geles. De hecho, se usa en las plantas cementeras ya que es un buen estabilizador y un agente hidrofilico, también es un buen espesante y un agente "suspensor" $y$ coloide protector. Su función en este nuevo material es el de ofrecer resistencia $y$, como también se reporta, para mejorar el brillo ya que es un aglutinante [29]. Se muestra también el otro agente, el almidón de tapioca comparado con los de maíz y trigo [30,31].

\subsubsection{Análisis de espectroscopía de infrarrojo (IR)}

Las muestras se caracterizaron por espectroscopía de infrarrojo (IR) para ver la homogeneidad de las bioespumas
- bioesponjas en toda la superficie. La espectroscopia infrarroja consiste en dar energía del orden de infrarrojo a las moléculas y obtener vibraciones de los enlaces los cuales proporcionan información de los grupos funcionales presentes [32]. El análisis se realizó en diferentes porciones de las muestras de bioesponja (hechas en cajas de Petri de $5 \mathrm{~cm}$ de diámetro). El gráfico de la Figura 5 muestra la mezcla de Q-Qn adicionada con los dos agentes plastificantes (CMC-AlmTap), en una relación 10,20,30 a $40 \%$ de ellos. En ella, la banda presente en 3358.94 $\mathrm{cm}^{-1}$ se compara con bandas 3434 y $3444 \mathrm{~cm}^{-1}$, que corresponden al grupo-OH [22].

También se puede observar la banda en $3434.24 \mathrm{~cm}^{-1}$ para el grupo $-\mathrm{OH}$, que pertenece a la quitina. La banda en $1629 \mathrm{~cm}^{-1}$ es característica del grupo $-\mathrm{NH}_{2}$, cuando está ligado al polímero de quitosana suele estar presente en $1621 \mathrm{~cm}^{-1}$, en la Figura 5 parece estar desplazado hasta la banda $1650.78 \mathrm{~cm}^{-1}$ [22]. 


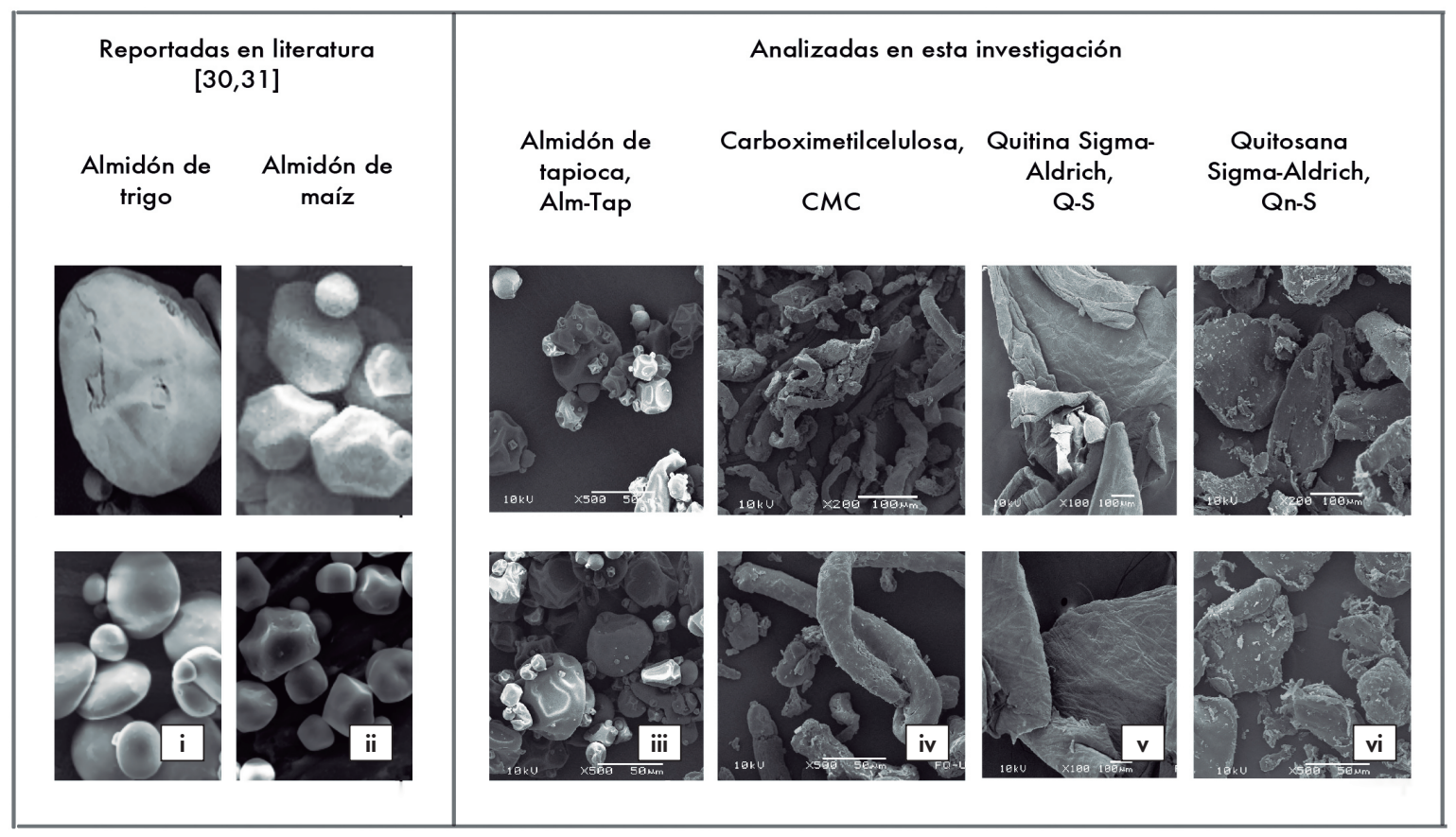

Figura 4. Microscopía electrónica de barrido de las diferentes materias primas y controles empleados en esta investigación y de dos almidones de (i) trigo y de (ii) maíz

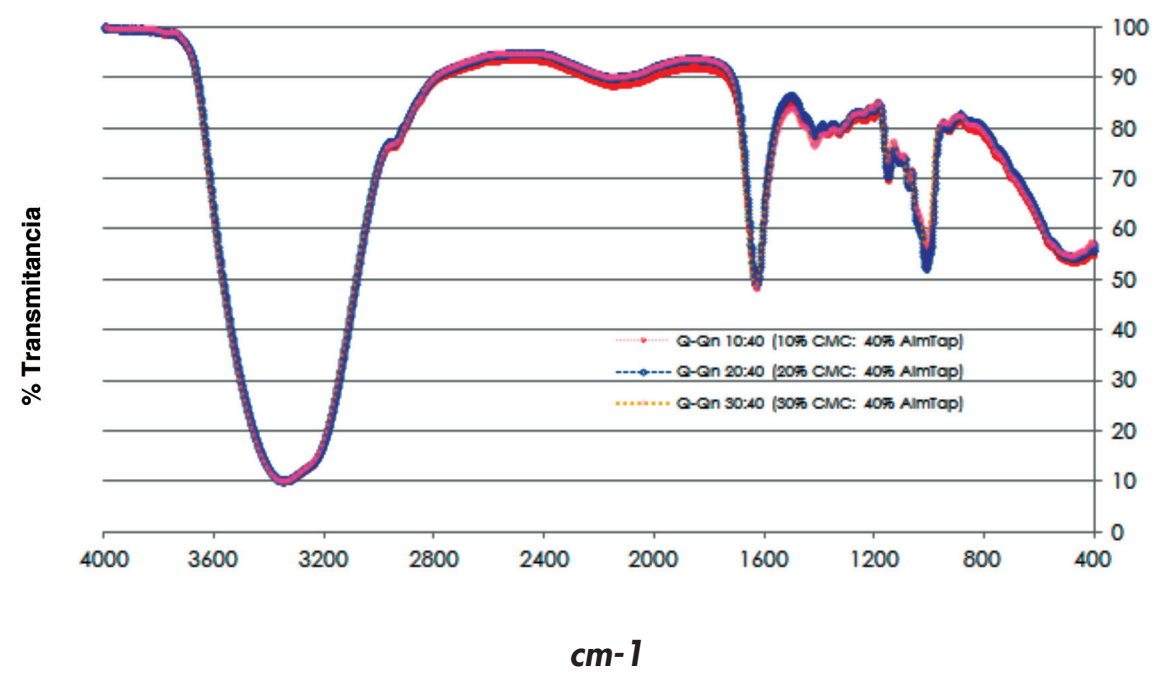

Figura 5. Espectro de infrarrojo, IR, para las bioespumas o bioesponjas hechas con la mezcla de agentes plastificantes 10, 20 y 30\% CMC y 40\% almidón de tapioca obtenidas de los residuos de camarón

\subsection{Análisis mecánicos de las bioespumas 0 bioesponjas}

La Tabla 5 presenta los valores del Módulo de Young en MPa para las probetas de quitina-quitosana con la mezcla de los dos agentes plastificantes $10 \%$ CMC, carboximetilcelulosa y $40 \%$ Alm-Tap, almidón de ta- pioca) obtenidos a partir de los datos experimentales del equipo Instron y del equipo de pruebas triaxiales cíclicas no confinadas. Se tomaron solamente las muestras que contienen CMC al 10\% y almidón de tapioca al $40 \%$ ya que son las que más se asemejan al unicel (muestras de poliuretano expandido). El análisis estadístico de estos datos $(p<0.05)$ indica que sí 
hay diferencia significativa por lo que sigue habiendo una oportunidad de mejora para continuar con los estudios de adición de otros agentes plastificantes (Figura 6), aunque debe mencionarse que las bioespumas 0 bioesponjas tienen mejores características que el unicel según los resultados de algunas pruebas subjetivas realizadas con jueces no entrenados sobre sus propiedades mecánicas ya que fueron menos deformables que los de poliestireno espumado [7].

Un ejemplo de las curvas obtenidas en uno de los equipos, el de pruebas triaxiales cíclicas no confinadas (compresión simple), se presentan en la Figura 7. En ella se tienen las pruebas mecánicas de compresión para las tres probetas obtenidas con los polímeros naturales provenientes de los residuos de camarón mezclados con los dos agentes plastificantes en una relación 10:40 (10\% CMC y 40\% Alm-Tap). En la Figura 8 se presenta la secuencia fotográfica en el equipo Instron.

El comportamiento elástico que presenta una probeta de poliestireno expandido al ser deformada puede verse con estudios previos realizados por Flores en 2008 [6]. En esa investigación las microfotografías que se tomaron fueron ya del sólido celular (bioespuma o bioesponja) que muestra cómo al ser un material biodegradable, el comportamiento estructural es diferente al del poliestireno espumado y al poliuretano espumado.

Tabla 5. Comparación de los valores del Módulo de Young en MPa para las tres probetas de quitina-quitosana con la mezcla de los dos agentes plastificantes (10\% carboximetilelulosa y 40\% almidón de tapioca) en el equipo Instron y el equipo de pruebas triaxiales cíclicas no confinadas y los datos para la muestra control de poliestireno espumado (unicel) de dos diferentes densidades

\begin{tabular}{|c|c|c|c|c|}
\hline \multirow{2}{*}{$\begin{array}{c}\text { Pruebas en los equipos } \\
\text { Número de replicas }\end{array}$} & \multicolumn{2}{|c|}{ Mezcla de agentes plastificantes [\%] } & \multicolumn{2}{|c|}{ Módulo de Young [MPa] } \\
\hline & CMC & AlmTap & Instron & Pruebas triaxiales cíclicas \\
\hline 1 & 10 & 40 & 0.4418 & 0.440 \\
\hline 2 & 10 & 40 & 0.5212 & 0.531 \\
\hline 3 & 10 & 40 & 0.5632 & 0.566 \\
\hline Promedio & & & $0.5087 \pm 0.0617$ & $0.535 \pm 0.065$ \\
\hline 1 & 20 & 40 & 1.416 & 0.487 \\
\hline 2 & 20 & 40 & 1.599 & 0.669 \\
\hline 3 & 20 & 40 & 1.550 & 0.424 \\
\hline Promedio & & & $1.521 \pm 0.094$ & $0.556 \pm 0.127$ \\
\hline 1 & 30 & 40 & 1.614 & 0.669 \\
\hline 2 & 30 & 40 & 1.542 & 0.733 \\
\hline 3 & 30 & 40 & 1.405 & 0.565 \\
\hline \multirow[t]{2}{*}{ Promedio } & & & $1.520 \pm 0.106$ & $0.694 \pm 0.084$ \\
\hline & \multicolumn{2}{|c|}{ Densidad $[\mathrm{g} / \mathrm{mL}]$} & & \\
\hline Unicel & \multicolumn{2}{|c|}{0.035} & $7.832^{*}$ & $1.858^{*}$ \\
\hline Unicel & \multicolumn{2}{|c|}{0.018} & $4.555^{\star}$ & $4.546^{*}$ \\
\hline
\end{tabular}

*No se hicieron triplicados debido a que la barra o "viga" de unicel de donde se tomó la muestra presentó homogeneidad por lo que se consideró innecesario 


\section{Means and 95.0 Percent LSD Intervals}

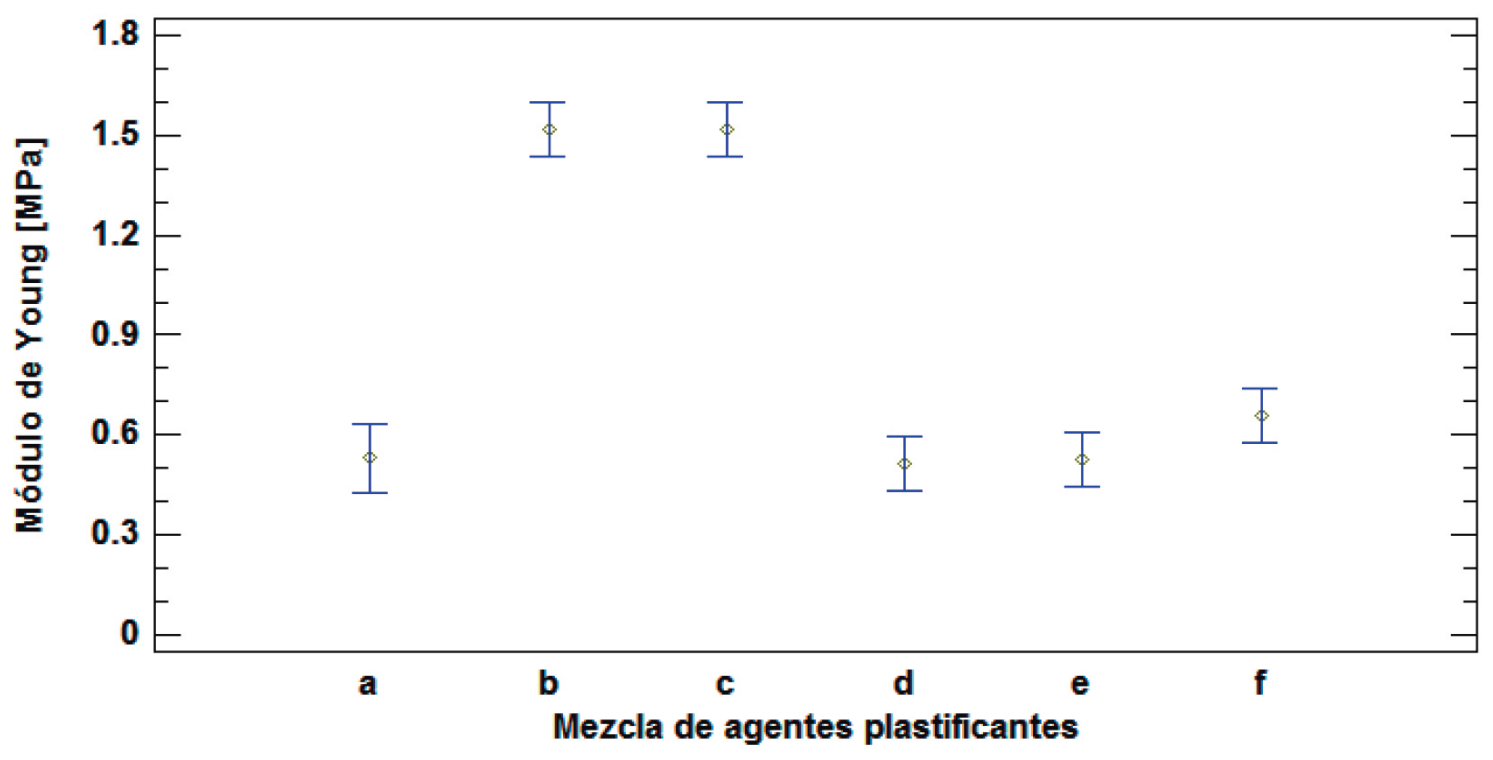

Figura 6. Resultados estadísticos de las diferencias de medias entre los datos de la Tabla 5. a, b y $\mathbf{c}$ con el equipo Instron; $\boldsymbol{d}$, e y $\boldsymbol{f}$ pruebas triaxiales cíclicas.

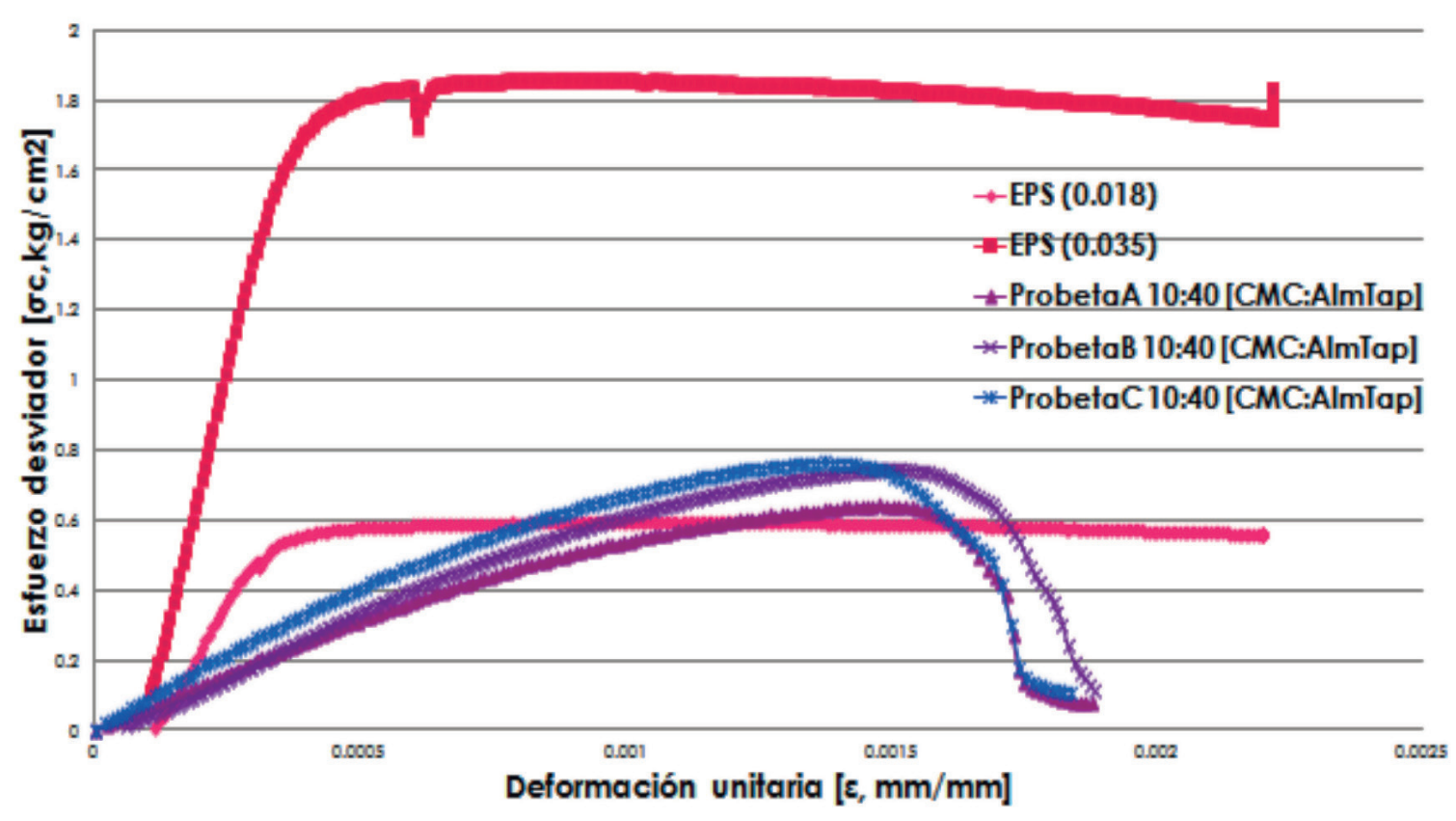

Figura 7. Módulo de Young para las probetas obtenidas con los residuos de camarón usando dos agentes plastificantes en una relación 10:40 (CMC:Alm-Tap) comparada con los controles de unicel (EPS) con dos diferentes densidades $(0.018$ y $0.032 \mathrm{~g} / \mathrm{mL})$ 


\begin{tabular}{|l|l|l|}
\hline Probeta antes de la prueba & Durante la prueba & $\begin{array}{c}\text { Probeta al momento } \\
\text { de la fractura }\end{array}$ \\
\hline $10: 40$ & & \\
\hline
\end{tabular}

Figura 8. Secuencia fotográfica para la fractura de las probetas obtenidas en esta investigación en el equipo Instron. Probetas de Q-Qn-10CMC-40AlmTap.

\section{Conclusiones}

De acuerdo con el objetivo de esta investigación de realizar la caracterización mecánica de los productos espumados obtenidos de los residuos sólidos del camarón utilizando agentes plastificantes para su posible aplicación en la ingeniería ambiental, puede concluirse lo siguiente:

El material polimérico natural proveniente de los exoesqueletos y cabezas de camarón, una mezcla de quitinaquitosana, Q-Qn, adicionado con una mezcla de los dos agentes plastificantes biodegradables en estudio carboximetilcelulosa y almidón de tapioca en una proporción de 10 y $40 \%$, respectivamente, fue el que mostró las mejores características mecánicas. Al comparar los resultados de los Módulos de Young obtenidos de estas bioespumas o bioesponjas con un polímero derivado del petróleo conocido en México como unicel, sus probetas fueron estadísticamente diferentes $(p<0.05)$, pero en pruebas subjetivas con jueces no entrenados [7] se consideraron mejores para almacenar alimentos por tener una menor "deformabilidad" que el poliestireno espumado.

\section{Reconocimiento}

El primer autor agradece al Consejo Nacional de Ciencia y Tecnología de México por la beca de maestría para realizar esta investigación. Los autores agradecen al personal académico de la Facultad de Ingeniería y el Instituto de Ingeniería de la UNAM por su valioso apoyo para realizar las pruebas mecánicas en sus Laboratorios de Materiales y Manufactura y de Geotecnia. 


\section{Referencias}

[1] SEMARNAT. 2013. Residuos. Secretaria de Medio Ambiente y Recursos Naturales. Última revisión. 28 de julio del 2013. Disponible en internet: [http://app1.semarnat.gob.mx/dgeia/informe_12/pdf/Cap7_residuos.pdf]

[2] INEGI. 2012. Conociendo México. Instituto Nacional de Estadística y Geografía. Última revisión. 28 de julio del 2013. Disponible en internet: [http://www.inegi.org.mx/prod_serv/contenidos/espanol/bvinegi/productos/integracion/pais/mexcon/folleto_nacional_pliegos_baja.pdf]

[3] Navarrete-Gatica, M.R. 2005. Disposición final de residuos sólidos municipales. Tesis de Licenciatura, UNAM, Facultad de Ingeniería. México, D. F. México. Recuperado el 11 de mayo de 2015, de sitio Web de la UNAM: http://132.248.9.195/pdtestdf/03444453/Index.html

[4] CONAPESCA. 2012. Comisión Nacional de Acuacultura y Pesca. Disponible en las Redes Internacionales (internet). Recuperado el 12 de noviembre del 2010, de sitio Web: [http://www.conapesca.sagarpa.gob.mx/wb/cona/anuario_2009_capitulo_i_preliminar]

[5] Flores, R.A. 2004. Bioplástico de quitina: Formación de películas de quitina a partir de desechos de camarón por métodos ecológicos. Tesis de Maestría en Ciencias. Programa de Maestría y Doctorado en Ciencias Químicas, UNAM, Facultad de Química. México, D. F. México. Recuperado el 11 de mayo de 2015, de sitio Web de la UNAM: http://132.248.9.195/ppt2004/0333631/lndex.html

[6] Flores, R.A. 2008. Obtención y caracterización de esponja de quitina a partir de cefalotórax de camarón. Tesis de Doctorado en Ciencias. Programa de Maestría y Doctorado en Ciencias Químicas, UNAM, Facultad de Química. México, D. F. México. Recuperado el 11 de mayo de 2015, de sitio Web de la UNAM: http://132.248.9.195/ptd2008/agosto/0629854/Index.html

[7] Ortega-Granados, J.A. 2014. Caracterización mecánica de productos espumados obtenidos de residuos sólidos de las pesquerías para su posible aplicación en la ingeniería ambiental. Tesis de Maestría en Ingeniería (Campo del conocimiento: Ingeniería Ambiental, Campo disciplinario: Sustancias y Residuos Peligrosos). Programa de Maestría y Doctorado en Ingeniería. UNAM. Agosto 15. México, D. F. México. Recuperado el 11 de mayo de 2015, de sitio Web de la UNAM: [http://oreon.dgbiblio.unam.mx/F/ME2L9H7M7ARVYNTG32ATMELSGF3P4YBMJ3A96B79E9VFKR14F5-46421 ?func=full-set-set\&set_number=036199\&set_entry=000001\&format=999]

[8] Ortega-Granados, J.A. 2011. Efecto del recubrimiento de fresas usando quitina-quitosana obtenida de cefalotórax y exoesqueleto de camarón en su vida de anaquel a temperatura ambiente $\left(20 \pm 2^{\circ} \mathrm{C}\right)$ y refrigeración $\left(4^{\circ} \mathrm{C}\right)$. Tesis de Licenciatura, UNAM, Facultad de Química. Noviembre 29. México, D. F. México. Recuperado el 11 de mayo del 2015 de sitio Web de la UNAM: [http://132.248.9.195/ptd2012/ mayo/0679804/Index.html]

[9] Pillai, C.K.S., Paul, W., Sharma C.P. 2009. Chitin and chitosan polymers: Chemistry, solubility and fiber formation. Progress in Polymer Science. 34(7):641-678.

[10] Salas-Osornio, J. 2011. Aprovechamiento integral de residuos crustáceos: evaluación de los recubrimientos de frutas frescas usando mezclas de quitina y quitosana obtenidas por medio de química verde. Tesis de Licenciatura, UNAM, Facultad de Química. México, D. F. México. Recuperado el 11 de mayo del 2015 de sitio Web de la UNAM: [http://132.248.9.195/ptd2012/marzo/0677848/Index.html]

[11] Shirai-Matsumoto, K. 2011. Producción de quitina y quitosana: Nuevo proceso biotecnológico para la obtención de quitina y quitosana. Pub. Universidad Autónoma Metropolitana. Campus Iztapalapa. México, D. F. México.

[12] Gutiérrez-Cabello, M., García-Gómez, R.S., Sánchez-Zamora, A., Suárez-Bautista, J., Gaxiola-Cortés, G., Chel-Guerrero, L., Duránde-Bazúa, C. 2009. Aprovechamiento integral de especies acuícolas del sureste de México: Beneficios obtenidos por la pigmentación 
"natural" de su carne / Global reuse of Mexico's southeast aquacultural species: Benefits obtained by its flesh "natural" pigmentation. Gutiérrez-Cabello, Carnilac Industrial. Edición Especial de la Industria Cárnica 2009:32-36.

[13] Majeti, N. V., Kumar, R. 2000. A review of chitin and chitosan applications. Reactive \& Functional Polymers, 46:1-27.

[14] Santosa, S.J., Siswanta, D., Sudiono, S., Utarianingrum, R. 2008. Chitin-humic acid hybrid as adsorbent for Cr (III) in effluent of wastewater treatment. Applied Surface Science, 254:7846-7850.

[15] Flores, R., Barrera-Rodríguez, S., Shirai, K., Durán-de-Bazúa, C. 2007. Chitin sponge, extraction procedure from shrimp wastes using green chemistry. J. Appl. Polym. Sci. 104:3909-3916.

[16] Flores, R., Barrera-Rodríguez, S., Shirai-Matsumoto, C.K., Durán-de-Bazúa, C. 2006. Obtención de esponjas de quitina a partir de cefalotórax de camarón para empaques. AlimenPack, 2(4):26-28.

[17] Arias-Torres, O., Mendoza-Márquez, A.M., Bárcenas-Ochoa, E.M., Flores, R., García-Gómez, R.S., Durán-Domínguez-de-Bazúa, M.C. 2013. Comparación de diferentes agentes plastificantes adicionados en esponjas de quitina obtenidas de cefalotórax de camarón. Revista Química Central de la Facultad de Ciencias Químicas (Ecuador). 3(1):11-18.

[18] Barrera-Rodríguez, S., Flores-Ortega, R.A., Durán-Domínguez-de-Bazúa, M.C. 2011. Extracción de quitina del cefalotórax de camarón para la elaboración de películas / Chitin extraction from shrimp cephalothorax and exoskeleton for films preparation. Vol. 10, Serie: TECNOLOGÍAS más LIMPIAS. Pub. AMCATH y Laboratorios 301, 302, 303 de Ing. Quím. Amb. y de Quím. Amb. Facultad de Química, UNAM. ISBN 978-607-7807-08-0. 75 págs. $1^{\text {a }}$ Ed. Disco compacto (2006). $2^{\text {a }}$ Ed. Mejorada, disco compacto (2007). $3^{\text {a }}$ Ed. con ISBN (2011). México D.F. México.

[19] Tomás-Reyes, S. 2015. Elaboración de bioesponjas de quitina extraída del cefalotórax y exoesqueleto de camarón mediante la utilización de plastificantes naturales, almidón de maíz. Tesis de Licenciatura, UNAM, Facultad de Química. México, D. F. México. Recuperado el 11 de mayo del 2015 de sitio Web de la UNAM: [http://132.248.9.195/ptd2014/noviembre/304159855/Index.html]

[20] Valencia-Ávila, I. 2012. Efecto de agentes plastificantes naturales en las propiedades mecánicas de esponjas de quitina. Tesis de Licenciatura, UNAM, Facultad de Estudios Profesionales Zaragoza. México, D. F. México. Recuperado el 11 de mayo del 2015 de sitio Web de la UNAM: [http://132.248.9.195/ptd2012/junio/300842018/Index.html]

[21] Durán-Domínguez-de-Bazúa, M.C., Barrera-Rodríguez, S., Flores-Ortega, R.A. 2004. Extracción ecológica de quitina y subproductos. Solicitud de Registro: Octubre 1, 2004. UNAM, Facultad de Química. IMPI. PA/a/2004/009517. Patente Núm. 264482. Otorgada el 12 de febrero de 2009. México D.F. México.

[22] Sarabia-Bañuelos, P. 2011. Aprovechamiento integral de residuos de crustáceos: obtención de quitina y quitosana del cefalotórax de camarón por métodos ecológicos. Tesis de Maestría en Ciencias. Programa de Maestría y Doctorado en Ciencias Químicas, UNAM, Facultad de Química. México, D. F. México. Recuperado el 11 de mayo del 2015 de sitio Web de la UNAM: [http://132.248.9.195/ ptd2012/enero/0676305/Index.html]

[23] Ortega-Granados, J.A., Durán-Domínguez-de-Bazúa, M.C. 2014. Proceso de química verde para la obtención de materiales espumados a partir de residuos sólidos de animales artrópodos y productos obtenidos del mismo. Solicitud de Registro: Diciembre 10, 2014. UNAM, Facultad de Química. IMPI. Folio MX/e/2014/088656. Expediente: MX/a/2014/015119. En proceso de otorgamiento. México D.F. México.

[24] Bárcenas-Ochoa, E.M. 2010. Biopolímeros de cefalotórax y exoesqueleto de camarón. Uso de aditivos químicos para modificar sus propiedades mecánicas. Tesis de Licenciatura, UNAM, Facultad de Química. Agosto 23. México, D. F. México. Recuperado el 11 de mayo del 2015 de sitio Web de la UNAM: [http://132.248.9.195/ptb2010/agosto/0660690/Index.html]

[25] Mendoza-Márquez, A.M., Arias-Torres, O. 2010. Caracterización mecánica de un polímero de origen natural derivado de cefalotórax y exoesqueletos de camarón. Tesis de Licenciatura, UNAM, Facultad de Química. Mayo 12. México, D. F. México. Recuperado el 11 de mayo del 2015 de sitio Web de la UNAM: [http://132.248.9.195/ptd2010/abril/0656717/Index.html]

[26] González-Mancera, G., Noguez-Amaya, M.E. 2006. Principios de microscopía electrónica de barrido y microanálisis por rayos X. Departamento de Ingeniería Química Metalúrgica. Facultad de Química, UNAM. ISBN 970-3-4011-9. Pp. 1-97. México D.F. México.

[27] Manahan, S.E. 2007. Introducción a la química ambiental. Coedición UNAM-Editorial Reverté. Barcelona-México D.F. España-México.

[28] Núñez-Bretón, L. C. 2009. Importancia del almidón (aspectos fisicoquímicos, usos y aplicaciones). Tesis profesional. UNAM, Facultad de Química. México, D. F. México.

[29] Sánchez-Soto, A. 2013. Caracterización de películas biodegradables y su aplicación como envase activo en zarzamora (Rubus frocticosus) para el control de podredumbre gris. Tesis profesional. UNAM, Facultad de Estudios Superiores Cuautitlán, México, D. F. México.

[30] Barrera, N.G., Calderón-Domínguez, G., Chanona-Pérez, J., Gutiérrez-López, G.F., León, A.E., Ribotta, P.D. 2013. Evaluation of the mechanical damage on wheat starch granules by SEM, ESEM, AFM and texture image analysis. Carbohydrate Polymers. 08:1449-1457.

[31] Sujka, M., Jamroz, J. 2013. Ultrasound-treated starch: SEM and TEM imaging, and functional behaviour. Food Hydrocolloids. $31: 413$ 419.

[32] Martínez-Hernández., N.B., Vásquez-Escobar, M. 2009. Obtención y caracterización de un material polimérico a partir de la mezcla de polietileno de baja densidad (PEBD) y almidón de maíz modificado. Tesis profesional. Universidad Veracruzana, Facultad de Ciencias Químicas. Campus Coatzacoalcos, México. Recuperado el 11 de mayo del 2015 de sitio Web de la UV: [http://cdigital.uv.mx/bitstream/12345678/938/1/Miriam\%20Vazquez\%20Escobar.pdf] 